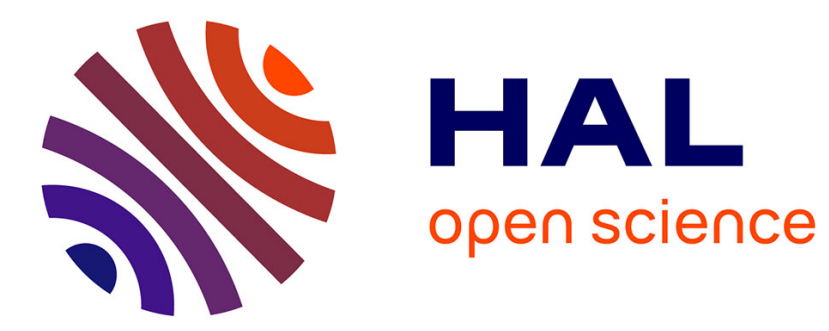

\title{
Multidimensional corrections to cell-centered finite volume methods for Maxwell equations
}

Brigitte Bidégaray, Jean-Michel Ghidaglia

\section{To cite this version:}

Brigitte Bidégaray, Jean-Michel Ghidaglia. Multidimensional corrections to cell-centered finite volume methods for Maxwell equations. Applied Numerical Mathematics, 2003, 44 (3), pp.281-298. 10.1016/S0168-9274(02)00171-X . hal-00319992

\section{HAL Id: hal-00319992 \\ https://hal.science/hal-00319992}

Submitted on 9 Jun 2021

HAL is a multi-disciplinary open access archive for the deposit and dissemination of scientific research documents, whether they are published or not. The documents may come from teaching and research institutions in France or abroad, or from public or private research centers.
L'archive ouverte pluridisciplinaire $\mathbf{H A L}$, est destinée au dépôt et à la diffusion de documents scientifiques de niveau recherche, publiés ou non, émanant des établissements d'enseignement et de recherche français ou étrangers, des laboratoires publics ou privés. 


\title{
Multidimensional corrections to cell-centered finite volume methods for Maxwell equations
}

\author{
B. Bidégaray ${ }^{1}$, J.-M. Ghidaglia ${ }^{2}$
}

\section{Introduction}

Most numerical computations of electromagnetic phenomena only involve monochromatic solutions which are described by the Helmholtz equation. This equation is a time-independent elliptic system and finite difference or finite element methods are particularly appropriate in this context. New applications for numerical simulations now necessitate the computation of solutions to the full time-dependent Maxwell equations. This is the case in the context of stealth for military aircrafts (more precisely the determination of the Radar Cross Section of the airship) but also in plasma physics and nonlinear electromagnetism in general.

Since Maxwell equations is a conservative system of hyperbolic equations, the finite volume methods are (with conservative finite difference methods) appropriate methods. Of course in the case of unstructured meshes, it appears that finite volume methods are more tractable. Again in the context of stealth, the industry tries to use RAM (radar absorbent material) coating. Since we are dealing with thin layers on the aircraft, it is clear that one must use a method that takes into account complex geometries like finite volume methods do. Moreover meshes corresponding to these geometries are obtained through a CAD software and they are also used to compute the

\footnotetext{
${ }^{1}$ Mathématiques pour l'Industrie et la Physique, CNRS UMR-5640, Université Paul Sabatier, 118 route de Narbonne, 31062 Toulouse Cedex 4, France. Permanent address: Laboratoire de Modélisation et de Calcul — IMAG, CNRS UMR-5523, B.P. 53, 38041 Grenoble Cedex 9, France.

${ }^{2}$ Centre de Mathématiques et de Leurs Applications, CNRS UMR-8536, Ecole normale supérieure de Cachan, 61 Avenue du Président Wilson, 94235 Cachan Cedex, France
} 
equation of fluid mechanics (CFD), see e.g. Schnack et al. [22] and Shankar et al. [23].

This gives at least two reasons for deriving finite volume method for the time-dependent Maxwell equations: on the one hand the hyperbolic character of the system is taken into consideration and on the other hand electromagnetic codes may be implemented using data structures initially designed for finite volume computational codes for the equations of fluid mechanics (Euler, Navier-Stokes,... ). This approach also allows to consider a possible coupling.

Although the above-mentioned applications are quite complex, there are still some very basic problems to solve for the simulation of the Maxwell equations. This article is focused on one of them, namely the fact that finite volume solutions to Maxwell equations often show some mesh dependent structures. This is due to the fact that solutions are piece-wise constant and wave equations well propagate discontinuites. Besides finite volume methods compute fluxes across edges in definite directions, increasing the directional effects, whereas the propagation of the physical wave is isotropic. To avoid this, some genuinely multidimensional approaches have been studied (see e.g. Lukáčová-Medviďová, Morton and Warnecke [18, 19, 20]). Our goal is to combine classical characteristic methods with multidimensional corrections. These corrections are derived following a method suggested by one of the author (JMG) and has led to $[13,14]$ and $[5]$ in the context of gas dynamics (later and independently Abgrall [1] has used the same type of ideas). It consists in computing the exact solution to the wave equation associated to piece-wise constant initial data on a given mesh.

The outline of this article is the following. In Section 1, we recall what cell-centered finite volumes are and fix our notations. In Section 2, we give the theory for the derivation of multidimensional corrections (the details of the effective computations are given in [2]) for first order and higher order methods. In Section 3, we describe the derivation of different boundary conditions.

Some partial results, with numerical results on simple test cases, have been announced in [3]. 


\section{Cell-centered finite volume methods for Maxwell equations}

The corrections we derive are specifically designed for cell-centered finite volume schemes, for which all the variables are discretized at the same location. It is necessary to introduce here the schemes we deal with, although they are not new, especially to describe notations which are also used in the expressions for the corrections.

\subsection{Formulations for Maxwell equations}

In their initial formulation Maxwell equations read

$$
\left\{\begin{aligned}
\frac{\partial \mathbf{D}}{\partial t}-\operatorname{curl} \mathbf{H} & =-\mathbf{j} \\
\frac{\partial \mathbf{B}}{\partial t}+\operatorname{curl} \mathbf{E} & =0 \\
\operatorname{div} \mathbf{D} & =\rho \\
\operatorname{div} \mathbf{B} & =0
\end{aligned}\right.
$$

together with the constitutive laws

$$
\mathbf{D}=\varepsilon \mathbf{E}, \quad \mathbf{B}=\mu \mathbf{H} .
$$

We are interested in the special case of vanishing charge and current densities. i.e. $\rho=0$ and $\mathbf{j}=0$. To design the method we use Maxwell equations in terms of variables $\mathbf{D}$ and $\mathbf{B}$ only, namely:

$$
\left\{\begin{array}{l}
\frac{\partial \mathbf{D}}{\partial t}-\operatorname{curl}(\mathbf{B} / \mu)=0 \\
\frac{\partial \mathbf{B}}{\partial t}+\operatorname{curl}(\mathbf{D} / \varepsilon)=0
\end{array}\right.
$$

Setting $\mathbf{U}={ }^{t}(\mathbf{D}, \mathbf{B})$ the conservative formulation for this system reads

$$
\frac{\partial \mathbf{U}}{\partial t}+\operatorname{div} \mathbb{A} \mathbf{U}=0
$$

where $\mathbb{A}$ is given in the next subsection. Physical boundary conditions will however be given in Section 3 in terms of variables $\mathbf{E}$ and $\mathbf{H}$. 


\subsubsection{TE and TM modes}

As we restrict the study to a two-dimensional space, we may decouple some of the variables. For instance if the space variable $z$ plays no role, the $\mathrm{TE}_{z}$ mode only deals with variables $\left(D_{x}, D_{y}, B_{z}\right)$ and the $\mathrm{TM}_{z}$ mode only with variables $\left(D_{z}, B_{x}, B_{y}\right)$. Since passing from one mode to another (in absence of charge current) is straightforward we restrict the study to the $\mathrm{TE}_{z}$ mode and therefore we take $\mathbf{U}={ }^{t}\left(D_{x}, D_{y}, B_{z}\right)$ and $\frac{\partial \mathbf{U}}{\partial t}+\operatorname{div} \mathbb{A} \mathbf{U}=0$ where $\mathbb{A}=\left(A_{x}, A_{y}\right)$ and

$$
A_{x}=\left(\begin{array}{ccc}
0 & 0 & 0 \\
0 & 0 & 1 / \mu \\
0 & 1 / \varepsilon & 0
\end{array}\right), A_{y}=\left(\begin{array}{ccc}
0 & 0 & -1 / \mu \\
0 & 0 & 0 \\
-1 / \varepsilon & 0 & 0
\end{array}\right) .
$$

\subsubsection{Finite volume formulation}

Given a space decomposition $\mathcal{T}$ that consists in polygonal cells $C_{i}$, we integrate equation (1.1) over one of these cells. We compute the evolution of $\mathbf{U}_{i}$ which is an approximate (mean) value of $\mathbf{U}$ on $C_{i}$. This reads

$$
\left|C_{i}\right| \frac{d \mathbf{U}_{i}}{d t}=-\sum_{j} \int_{C_{i j}} \mathbb{A} \mathbf{U} \cdot \mathbf{n} d \sigma=-\sum_{j} \boldsymbol{\Phi}_{i j},
$$

where the summation involves indices $j$ relative to the cells $C_{j}$ that have a common interface (edge) $C_{i j}$ with $C_{i}$, and where $\left|C_{i}\right|$ denotes the area of $C_{i}$ and $\mathbf{n}$ is the outgoing unit normal vector to $C_{i}$ pointing into $C_{j}$.

We notice that $\boldsymbol{\Phi}_{i j}=\left|C_{i j}\right| \widehat{\mathbb{A} \mathbf{U}} \cdot \mathbf{n}_{i j}$, where $\mathbf{n}_{i j}$ denotes the outgoing unit normal vector to $C_{i}$ across $C_{i j},\left|C_{i j}\right|$ is the length of the edge $C_{i j}$ and $\widehat{\mathbb{A} \mathbf{U}}$ is the mean value of $\mathbb{A} \mathbf{U}$ on the interface. We set $A(\mathbf{U}, \mathbf{n})=\mathbf{n} \cdot \nabla_{\mathbf{U}} \mathbb{A} \mathbf{U}$ that does not depend on $\mathbf{U}$ when dealing with linear Maxwell equations. Therefore we will write $A(\mathbf{n})=A(\mathbf{U}, \mathbf{n})$ in the sequel and $\Phi_{i j}=\left|C_{i j}\right| A\left(\mathbf{n}_{i j}\right) \mathbf{U}$.

The definition of the finite volume method is only determined by the approximation that is chosen for $\boldsymbol{\Phi}_{i j}$, i.e. the approximation $\widehat{A\left(\mathbf{n}_{i j}\right)} \mathbf{U}$ of $A\left(\mathbf{n}_{i j}\right) \mathbf{U}$ on the interface. 


\subsection{Cell-centered methods}

\subsubsection{Steger-Warming decomposition}

The Steger-Warming decomposition [24] consists in splitting the wave according to the different characteristics. The eigenvalues of matrix $A(\mathbf{n})$ are $\pm c$, where $c=(\varepsilon \mu)^{-1 / 2}$, and 0 . We rewrite matrix $A(\mathbf{n})$ as $A(\mathbf{n})=P \Lambda(\mathbf{n}) P^{-1}$ where $\Lambda(\mathbf{n})$ is a diagonal matrix the elements of which are the eigenvalues of $A(\mathbf{n})$. Then the splitting reads $A(\mathbf{n})=A^{+}(\mathbf{n})+A^{-}(\mathbf{n})$ with $A^{ \pm}(\mathbf{n})=$ $P \Lambda^{ \pm}(\mathbf{n}) P^{-1}$ and $\Lambda^{+}(\mathbf{n})$ (resp. $\Lambda^{-}(\mathbf{n})$ ) only contains the eigenvalue $c$ (resp. $-c$ ). To reduce notations we set $Z=\sqrt{\mu / \varepsilon}$ and $Y=Z^{-1}=\sqrt{\varepsilon / \mu}$. Once the calculations are made, one finds that

$$
A^{ \pm}(\mathbf{n})=\frac{1}{2}(A(\mathbf{n}) \pm|A|(\mathbf{n})) .
$$

Let us set $\mathbf{n}=\left(n_{x}, n_{y}\right)$ then

$$
A(\mathbf{n})=c\left(\begin{array}{ccc}
0 & 0 & -n_{y} Y \\
0 & 0 & n_{x} Y \\
-n_{y} Z & n_{x} Z & 0
\end{array}\right) \text { and } \Lambda(\mathbf{n})=\left(\begin{array}{ccc}
-c & 0 & 0 \\
0 & 0 & 0 \\
0 & 0 & c
\end{array}\right) .
$$

The change of basis is given by

$$
P=\left(\begin{array}{ccc}
n_{y} Y & n_{x} & -n_{y} Y \\
-n_{x} Y & n_{y} & n_{x} Y \\
1 & 0 & 1
\end{array}\right), P^{-1}=\frac{1}{2}\left(\begin{array}{ccc}
n_{y} Z & -n_{x} Z & 1 \\
2 n_{x} & 2 n_{y} & 0 \\
-n_{y} Z & n_{x} Z & 1
\end{array}\right)
$$

and therefore the "directional" matrices read

$$
A^{ \pm}(\mathbf{n})=\frac{c}{2}\left(\begin{array}{ccc} 
\pm n_{y}^{2} & \mp n_{x} n_{y} & -n_{y} Y \\
\mp n_{x} n_{y} & \pm n_{x}^{2} & n_{x} Y \\
-n_{y} Z & n_{x} Z & \pm 1
\end{array}\right)
$$

and

$$
|A|(\mathbf{n})=c\left(\begin{array}{ccc}
n_{y}^{2} & -n_{x} n_{y} & 0 \\
-n_{x} n_{y} & n_{x}^{2} & 0 \\
0 & 0 & 1
\end{array}\right)
$$

Hence the Steger-Warning flux $\boldsymbol{\Phi}_{i j}^{S W}$ consists in choosing

$$
A(\mathbf{n}) \mathbf{U}=A^{+}(\mathbf{n}) \tilde{\mathbf{U}}_{i}+A^{-}(\mathbf{n}) \tilde{\mathbf{U}}_{j} .
$$


In this expression $\tilde{\mathbf{U}}_{i}$ and $\tilde{\mathbf{U}}_{j}$ are approximate values for $\mathbf{U}_{i}$ and $\mathbf{U}_{j}$ which will be chosen below to ensure a certain order to the numerical method. Expression (1.4) means that everything that goes out of the cell may only depend on the value inside the cell and what enters the cell across a given edge only depends on the value in the neighboring cell. The corresponding Steger-Warming flux reads

$$
\boldsymbol{\Phi}_{i j}^{S W}\left(\tilde{\mathbf{U}}_{i}, \tilde{\mathbf{U}}_{j}\right)=\left|C_{i j}\right|\left(A^{+}(\mathbf{n}) \tilde{\mathbf{U}}_{i}+A^{-}(\mathbf{n}) \tilde{\mathbf{U}}_{j}\right)
$$

and

$$
\left|C_{i}\right| \frac{d \mathbf{U}_{i}}{d t}=-\sum_{j} \boldsymbol{\Phi}_{i j}^{S W}\left(\tilde{\mathbf{U}}_{i}, \tilde{\mathbf{U}}_{j}\right) .
$$

\subsubsection{First order method}

The simplest choice is to take $\tilde{\mathbf{U}}_{i}=\mathbf{U}_{i}$, i.e. the value that is taken into account is the one at the "center" of the cell. This leads to a first order approximation and is the classical upwind flux, which is a generalization of the one-dimensional flux of Lax, Harten and van Leer [16].

\subsubsection{Gradient reconstruction}

In order to design higher order methods (next paragraph), we once more use a Steger-Warming flux but it is not applied to the constant values in the cells anymore, but to evaluations of the value on each side of the interface, $\mathbf{U}_{i j}$ and $\mathbf{U}_{j i}$. This evaluation requires to construct a value for the gradient in each cell $C_{i}$ and we denote it by $\nabla \mathbf{U}_{i}$.

Given constant values in each cell, different reconstructions for the gradient may be thought of. We describe here a method that is based on a Green formula and therefore close to flux calculations in finite volume schemes. It has the advantage of simplicity and applies to any type of mesh. Other reconstructions may be found in the literature. They have been developed in the context of computational fluid dynamics and only apply to triangles in order to define Galerkin basis functions. They are either based on a reconstruction of the gradient at the nodes [9] or in the elements [11]. Their application to Maxwell equations is discussed in $[6,7,8,15]$. In [21] it is shown that the finite volume gradient formulation gives as good results as these more 
complicated formulations. The Green formula $\int_{C_{i}} \nabla \mathbf{U} d \mathbf{x}=\int_{\partial C_{i}} \mathbf{U} \cdot \mathbf{n} d \sigma$ leads to the approximation

$$
\nabla \mathbf{U}_{i}=\frac{1}{\left|C_{i}\right|} \sum_{j} \frac{\mathbf{U}_{i}+\mathbf{U}_{j}}{2}\left|C_{i j}\right| \mathbf{n}_{i j},
$$

where the sum involves neighboring cells to $C_{i}$.

\subsubsection{Higher order methods}

For accuracy and stability reasons, a $\beta$-scheme is used, which is given for $\beta \in] 0,1\left[\right.$ by $\boldsymbol{\Phi}_{i j}=\boldsymbol{\Phi}_{i j}^{S W}\left(\mathbf{U}_{i j}, \mathbf{U}_{j i}\right)$ and

$$
\begin{aligned}
& \mathbf{U}_{i j}=\mathbf{U}_{i}+\frac{1}{2}\left\{(1-2 \beta)\left(\mathbf{U}_{i}-\mathbf{U}_{j}\right)+2 \beta \nabla \mathbf{U}_{i} \cdot G_{i} G_{j}\right\}, \\
& \mathbf{U}_{j i}=\mathbf{U}_{j}-\frac{1}{2}\left\{(1-2 \beta)\left(\mathbf{U}_{i}-\mathbf{U}_{j}\right)+2 \beta \nabla \mathbf{U}_{j} \cdot G_{i} G_{j}\right\},
\end{aligned}
$$

where $G_{i}$ is the barycenter of the cell $C_{i}$. This is an interpolation between two ways to compute $\mathbf{U}_{i j}$ : the mean value $\mathbf{U}_{i j}=\mathbf{U}_{j i}=\left(\mathbf{U}_{i}+\mathbf{U}_{j}\right) / 2$, and the half-upwind gradient calculation

$$
\begin{aligned}
& \mathbf{U}_{i j}=\mathbf{U}_{i}+\frac{1}{2} \nabla \mathbf{U}_{i} \cdot G_{i} G_{j}, \\
& \mathbf{U}_{j i}=\mathbf{U}_{j}-\frac{1}{2} \nabla \mathbf{U}_{j} \cdot G_{i} G_{j} .
\end{aligned}
$$

The $\beta$-scheme is proved to be a third order scheme for the specific choice $\beta=\frac{1}{3}$ in the case of node Galerkin gradient reconstructions (see [10]).

We may of course think of other schemes, for example in [4] is introduced a $\beta$ - $\gamma$-scheme that consists in coupling the $\beta$-scheme with a relaxation of the definition for $A^{ \pm}$given by equation (1.3)

$$
A^{ \pm}(\mathbf{n})=\frac{1}{2}(A(\mathbf{n}) \pm \gamma|A|(\mathbf{n}))
$$

where $\gamma \in[0,1]$, in order to control numerical diffusion. Since we add corrections, we do not consider such schemes that follow an other philosophy for correcting computed solutions. 


\subsection{Time discretization}

In practice the finite volume formulation (1.2) is solved in time using an explicit scheme that has the same order than the space method. This is based on the following approximation

$$
\left.\frac{d}{d t} \int_{C_{i}} \mathbf{U} d \mathbf{x} \simeq \frac{d}{d t} \int_{C_{i}} \mathbf{U} d \mathbf{x}\right|_{t=0} \equiv-\Psi_{i}(\mathbf{U}),
$$

where the notation $\Psi_{i}(\mathbf{U})$ is only introduced to make simpler the description of the numerical schemes below.

For the first order approximation, there is no need to use something more elaborate than the simplest first order explicit scheme

$$
\mathbf{U}_{i}^{n+1}=\mathbf{U}_{i}^{n}-\frac{\Delta t}{\left|C_{i}\right|} \Psi_{i}\left(\mathbf{U}^{n}\right) .
$$

For the higher order approximation a third order Runge-Kutta method is typically implemented

$$
\begin{aligned}
& \mathbf{U}_{i}^{(0)}=\mathbf{U}_{i}^{n}, \\
& \mathbf{U}_{i}^{(l)}=\mathbf{U}_{i}^{(0)}-\frac{\Delta t}{(4-l)\left|C_{i}\right|} \Psi_{i}\left(\mathbf{U}^{(l-1)}\right), \quad l=1,2,3, \\
& \mathbf{U}_{i}^{n+1}=\mathbf{U}_{i}^{(3)} .
\end{aligned}
$$

\section{Multidimensional corrections}

The corrections we introduce in this paper consist in giving an exact value for the time derivative instead of the approximation (1.6). This exact value is easier to derive from the wave equation. Indeed, in the case when there is no charge and charge current, each component of $\mathbf{U}$ is solution to a wave equation. Integrating this equation over a cell $C_{i}$ and the time interval $[0, t]$, we obtain

$$
\frac{d}{d t} \int_{C_{i}} \mathbf{U} d \mathbf{x}=\left.\frac{d}{d t} \int_{C_{i}} \mathbf{U} d \mathbf{x}\right|_{t=0}+c^{2} \int_{\partial C_{i}} \frac{\partial}{\partial n}\left(\int_{0}^{t} \mathbf{U}(\sigma, s) d s\right) d \sigma
$$

The evaluation of the first term in the right hand-side will be done using one of the classical methods described in Section 1.2. To evaluate the second 
term, that we denote by $\mathbf{K}_{i}$, we use the fact that we know an exact solution to the wave equation, that is given by Kirchoff formulae. This explicit expression has already been used in scientific computation. We may cite articles by Gilquin et al. $[13,14]$ and the PhD thesis of S. Chaïra [5] in which an exact Riemann problem is solved using the above equations after having linearized the equations of gas dynamics to obtain a wave equation. The calculations have however to be reproduced for Maxwell equations since the solutions are not self-similar, a property extensively used in the gas dynamics context.

\subsection{Kirchoff formulae}

Let $u: \mathbb{R}^{2} \times \mathbb{R}^{+} \rightarrow \mathbb{R}$ be solution to the wave equation

$$
\begin{cases}\frac{\partial^{2}}{\partial t^{2}} u(\mathbf{x}, t)-c^{2} \Delta u(\mathbf{x}, t)=f(\mathbf{x}, t), & \mathbf{x} \in \mathbb{R}^{2}, t>0, \\ u(\mathbf{x}, 0)=u_{0}(\mathbf{x}), & \mathbf{x} \in \mathbb{R}^{2}, \\ u_{t}(\mathbf{x}, 0)=u_{1}(\mathbf{x}), & \mathbf{x} \in \mathbb{R}^{2} .\end{cases}
$$

The exact solution to the Cauchy problem (2.2) is given by Kirchoff formulae:

$$
u(\cdot ; t)=\frac{\partial}{\partial t} F(\cdot ; t) \star u_{0}+F(\cdot ; t) \star u_{1}+\int_{0}^{t} d s F(\cdot ; s) \star f(\cdot, s) .
$$

In equation (2.3) the convolution is performed with respect to the space variable $\mathbf{x}$ and

$$
F(\mathbf{x} ; t)=\frac{1}{2 \pi c} \frac{H\left(c^{2} t^{2}-|\mathbf{x}|^{2}\right)}{\sqrt{c^{2} t^{2}-|\mathbf{x}|^{2}}}
$$

is the fundamental solution to the wave equation, where $H$ is the Heaviside function. Equation (2.3) holds in the sense of distributions.

\subsection{Calculation of the correction}

\section{Proposition 1}

(i) For the first order model, let us define the set of neighboring edges to one specific edge $\partial C_{i}^{k}$ of cell $C_{i}: N_{i}^{k}=\left\{(j, l) / j \neq i, \partial C_{j}^{l} \cap \partial C_{i}^{k} \neq \emptyset\right\}$, then $\boldsymbol{K}_{i}$ 
reads

$$
\begin{aligned}
\boldsymbol{K}_{i}= & -\frac{c}{2 \pi} \sum_{k} \sum_{(j, l) \in N_{i}^{k}}\left(\left(\boldsymbol{U}_{0}\right)_{j}-\left(\boldsymbol{U}_{0}\right)_{i}\right)\left(\mathbf{n}_{j} \cdot \mathbf{n}_{i}\right) \mathcal{I}_{0}\left(\partial C_{i}^{k}, \partial C_{j}^{l}\right) \\
& -\frac{c t}{2 \pi} \sum_{k} \sum_{(j, l) \in N_{i}^{k}} A\left(\mathbf{n}_{j}\right)\left(\left(\boldsymbol{U}_{0}\right)_{j}-\left(\boldsymbol{U}_{0}\right)_{i}\right) \mathcal{I}_{1}\left(\partial C_{i}^{k}, \partial C_{j}^{l}\right) .
\end{aligned}
$$

This correction does not change the conservativeness of the finite volume scheme.

(ii) For the second order model, let us define the set of neighboring cells to one specific edge $\partial C_{i}^{k}$ of cell $C_{i}: M_{i}^{k}=\left\{j / C_{j} \cap \partial C_{i}^{k} \neq \emptyset\right\}$, then $\boldsymbol{K}_{i}$ reads

$$
\begin{aligned}
\boldsymbol{K}_{i}= & -\frac{c}{2 \pi} \sum_{k} \sum_{j \in M_{i}^{k}}\left(\nabla \boldsymbol{U}_{0}\right)_{j} \cdot \mathbf{n}_{i} \mathcal{J}_{0}\left(\partial C_{i}^{k}, C_{j}\right) \\
& +\frac{c t}{2 \pi} \sum_{k} \sum_{j \in M_{i}^{k}} \mathbb{A}\left(\nabla \boldsymbol{U}_{0}\right)_{j} \mathcal{J}_{1}\left(\partial C_{i}^{k}, C_{j}\right) .
\end{aligned}
$$

Each component of the vector $\mathbf{U}$ is solution to a wave equation (2.2). If we set $\mathbf{W}^{i}(\mathbf{x}, t)=\int_{0}^{t}\left(\mathbf{U}(\mathbf{x}, s)-\mathbf{U}_{i}(\mathbf{x}, 0)\right) d s$ that is defined on the whole space, $\mathbf{W}^{i}$ is also solution to a wave equation where $\mathbf{W}_{0}^{i}=0, \mathbf{W}_{1}^{i}=\mathbf{U}_{0}-\left(\mathbf{U}_{0}\right)_{i}$ (where $\left.\left.\mathbf{U}_{i}(\cdot, 0)\right)=\left(\mathbf{U}_{0}\right)_{i}\right), f^{i}=\mathbf{U}_{1}$. The variable $\mathbf{W}^{i}$ is used instead of $\mathbf{U}$ for computing the correction $\mathbf{K}_{i}$. The time integration makes it more regular and subtracting $\mathbf{U}_{i}$ consists in studying fluctuations of the variable and not its value. The correction $\mathbf{K}_{i}$ now reads

$$
\mathbf{K}_{i}=c^{2} \int_{\partial C_{i}} \frac{\partial}{\partial n}\left(\int_{0}^{t} \mathbf{U}(\sigma, s) d s\right) d \sigma=c^{2} \int_{\partial C_{i}} \frac{\partial}{\partial n} \mathbf{W}^{i}(\sigma, t) d \sigma .
$$

Then calculations will differ according to the fact that we have reconstructed a gradient (second or third order scheme) or not (first order scheme) in the treatment of the first term in equation (2.1). In the first order case, $\mathbf{U}_{1}$ is a Dirac function supported by the edges of the mesh. In the higher order case, $\mathbf{U}_{1}$ has a reconstructed constant value on each cell.

In the sequel indices for normal vectors and integration variables specify the triangulation element we refer to, which is crucial for determining signs. 
According to (1.1), $\mathbf{U}_{1}=-\operatorname{div} \mathbb{A}\left(\mathbf{U}_{0}-\left(\mathbf{U}_{0}\right)_{i}\right)$ and we compute for each index $i$ the correction

$$
\begin{aligned}
\mathbf{K}_{i} & =c^{2} \int_{\partial C_{i}} \nabla\left(F(\cdot ; t) \star\left(\mathbf{U}_{0}-\left(\mathbf{U}_{0}\right)_{i}\right)+\int_{0}^{t} d s F(\cdot ; s) \star \mathbf{U}_{1}\right)\left(\sigma_{i}\right) \cdot \mathbf{n}_{i} d \sigma_{i} \\
& =c^{2} \int_{\partial C_{i}}\left(F(\cdot ; t) \star \nabla\left(\mathbf{U}_{0}-\left(\mathbf{U}_{0}\right)_{i}\right)+\nabla \int_{0}^{t} d s F(\cdot ; s) \star \mathbf{U}_{1}\right)\left(\sigma_{i}\right) \cdot \mathbf{n}_{i} d \sigma_{i}
\end{aligned}
$$

where we notice that $\nabla\left(\mathbf{U}_{0}-\left(\mathbf{U}_{0}\right)_{i}\right)$ and $\mathbf{U}_{1}$ have the same "regularity".

In order to be able to write convolutions properly, we introduce the notation $G(\mathbf{x} ; t)$ for the kernel $\nabla \int_{0}^{t} d s F(\mathbf{x} ; s)$. We compute this kernel in terms of $F(\mathbf{x} ; t)$. Let us set $\mathbf{x}=(x, y), r=|\mathbf{x}|$. For $0<r \leq c t$

$$
\begin{aligned}
G(\mathbf{x} ; t) & =\left(\begin{array}{c}
x / r \\
y / r
\end{array}\right) \frac{\partial}{\partial r} g(r ; t), \\
g(r ; t) & =\frac{1}{2 \pi c} \int_{r / c}^{t} \frac{1}{\sqrt{c^{2} s^{2}-r^{2}}} d s=\frac{1}{2 \pi c^{2}} \operatorname{Argch}\left(\frac{c t}{r}\right), \\
\frac{\partial}{\partial r} g(r ; t) & =-\frac{t}{2 \pi r c} \frac{1}{\sqrt{c^{2} t^{2}-r^{2}}}=-\frac{t}{r} F(\mathbf{x} ; t), \\
G(\mathbf{x} ; t) & =-\frac{t \mathbf{x}}{|\mathbf{x}|^{2}} F(\mathbf{x} ; t) .
\end{aligned}
$$

This equality also holds true for $|\mathbf{x}|>c t$. Thus

$$
\mathbf{K}_{i}=c^{2} \int_{\partial C_{i}}\left(F(\cdot ; t) \star \nabla\left(\mathbf{U}_{0}-\left(\mathbf{U}_{0}\right)_{i}\right)+G(\cdot ; t) \star \mathbf{U}_{1}\right)\left(\sigma_{i}\right) \cdot \mathbf{n}_{i} d \sigma_{i}
$$

\subsubsection{First order case}

Exact value for the correction. In this case we have to get rid of all derivatives in front of $\mathbf{U}_{0}$ since they are not defined in a classical way. In the first place we have 


$$
\begin{aligned}
\left(F(\cdot ; t) \star \nabla\left(\mathbf{U}_{0}-\left(\mathbf{U}_{0}\right)_{i}\right)\right)\left(\sigma_{i}\right)= & \\
& =\sum_{j / C_{j} \in \mathcal{T}} \int_{C_{j}} F\left(\sigma_{i}-\mathbf{y} ; t\right) \nabla\left(\mathbf{U}_{0}(\mathbf{y})-\left(\mathbf{U}_{0}\right)_{i}\right) d \mathbf{y} \\
& =-\sum_{j / C_{j} \in \mathcal{T}}\left(\left(\mathbf{U}_{0}\right)_{j}-\left(\mathbf{U}_{0}\right)_{i}\right) \int_{\partial C_{j}} F\left(\sigma_{i}-\sigma_{j} ; t\right) \mathbf{n}_{j} d \sigma_{j}
\end{aligned}
$$

We notice that $\mathbb{A}\left(\mathbf{U}_{0}-\left(\mathbf{U}_{0}\right)_{i}\right)$ belongs to $\mathbb{R}^{2}$ and use in what follows the fact that for two vectors, $\alpha$ and $\beta$, which take their values in $\mathbb{R}^{2}$, the following relation holds: $\int \alpha(\nabla \cdot \beta)=-\int(\nabla \otimes \alpha) \beta$. Therefore

$$
\begin{aligned}
& \left(G(\cdot ; t) \star \mathbf{U}_{1}\right)\left(\sigma_{i}\right)=-t \int_{\mathbf{y} \in \mathbb{R}^{2}} \frac{\sigma_{i}-\mathbf{y}}{\left|\sigma_{i}-\mathbf{y}\right|^{2}} F\left(\sigma_{i}-\mathbf{y} ; t\right) \mathbf{U}_{1}(\mathbf{y}) d \mathbf{y} \\
& \quad=t \int_{\mathbf{y} \in \mathbb{R}^{2}} \frac{\sigma_{i}-\mathbf{y}}{\left|\sigma_{i}-\mathbf{y}\right|^{2}} F\left(\sigma_{i}-\mathbf{y} ; t\right) \operatorname{div}_{\mathbf{y}}\left(\mathbb{A}\left(\mathbf{U}_{0}(\mathbf{y})-\left(\mathbf{U}_{0}\right)_{i}\right)\right) d \mathbf{y} \\
& \quad=-t \sum_{j / C_{j} \in \mathcal{T}} \int_{C_{j}} \operatorname{div}_{\mathbf{y}} \otimes\left(\frac{\sigma_{i}-\mathbf{y}}{\left|\sigma_{i}-\mathbf{y}\right|^{2}} F\left(\sigma_{i}-\mathbf{y} ; t\right)\right) \mathbb{A}\left(\mathbf{U}_{0}(\mathbf{y})-\left(\mathbf{U}_{0}\right)_{i}\right) d \mathbf{y} \\
& \quad=-t \sum_{j / C_{j} \in \mathcal{T}}\left(\int_{\partial C_{j}} \mathbf{n}_{j} \otimes \frac{\sigma_{i}-\sigma_{j}}{\left|\sigma_{i}-\sigma_{j}\right|^{2}} F\left(\sigma_{i}-\sigma_{j} ; t\right) d \sigma_{j}\right) \mathbb{A}\left(\left(\mathbf{U}_{0}\right)_{j}-\left(\mathbf{U}_{0}\right)_{i}\right) .
\end{aligned}
$$

Hence collecting the two terms in (2.4)

$$
\begin{aligned}
\mathbf{K}_{i}= & -c^{2} \sum_{j / C_{j} \in \mathcal{T}} \int_{\partial C_{i} \times \partial C_{j}} d \sigma_{j} d \sigma_{i}\left[\left(\left(\mathbf{U}_{0}\right)_{j}-\left(\mathbf{U}_{0}\right)_{i}\right) F\left(\sigma_{i}-\sigma_{j} ; t\right) \mathbf{n}_{j}\right. \\
& \left.+t\left(\mathbf{n}_{j} \otimes \frac{\sigma_{i}-\sigma_{j}}{\left|\sigma_{i}-\sigma_{j}\right|^{2}} F\left(\sigma_{i}-\sigma_{j} ; t\right)\right) \mathbb{A}\left(\left(\mathbf{U}_{0}\right)_{j}-\left(\mathbf{U}_{0}\right)_{i}\right)\right] \cdot \mathbf{n}_{i} \\
= & -c^{2} \sum_{j / C_{j} \in \mathcal{T}} \int_{\partial C_{i} \times \partial C_{j}} d \sigma_{j} d \sigma_{i}\left[\left(\left(\mathbf{U}_{0}\right)_{j}-\left(\mathbf{U}_{0}\right)_{i}\right) F\left(\sigma_{i}-\sigma_{j} ; t\right) \mathbf{n}_{j} \cdot \mathbf{n}_{i}\right. \\
& \left.+t \mathbf{n}_{j} \cdot \mathbb{A}\left(\left(\mathbf{U}_{0}\right)_{j}-\left(\mathbf{U}_{0}\right)_{i}\right)\left(\frac{\sigma_{i}-\sigma_{j}}{\left|\sigma_{i}-\sigma_{j}\right|^{2}} F\left(\sigma_{i}-\sigma_{j} ; t\right) \cdot \mathbf{n}_{i}\right)\right]
\end{aligned}
$$




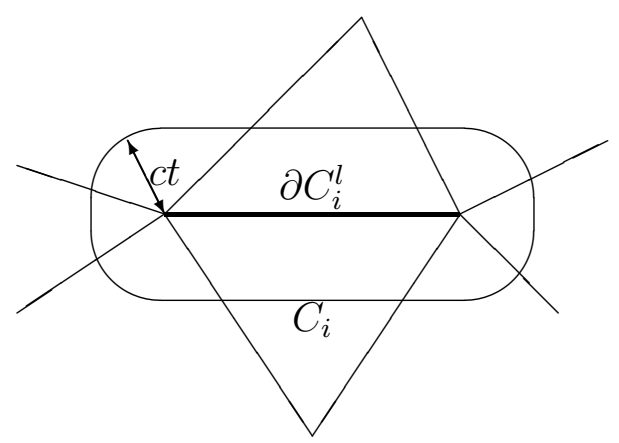

Figure 1: Influence over a time $t$

$$
\begin{aligned}
= & -c^{2} \sum_{j / C_{j} \in \mathcal{T}} \int_{\partial C_{i} \times \partial C_{j}} d \sigma_{j} d \sigma_{i}\left[\left(\left(\mathbf{U}_{0}\right)_{j}-\left(\mathbf{U}_{0}\right)_{i}\right) F\left(\sigma_{i}-\sigma_{j} ; t\right) \mathbf{n}_{j} \cdot \mathbf{n}_{i}\right. \\
& \left.+t A\left(\mathbf{n}_{j}\right)\left(\left(\mathbf{U}_{0}\right)_{j}-\left(\mathbf{U}_{0}\right)_{i}\right)\left(\frac{\sigma_{i}-\sigma_{j}}{\left|\sigma_{i}-\sigma_{j}\right|^{2}} F\left(\sigma_{i}-\sigma_{j} ; t\right) \cdot \mathbf{n}_{i}\right)\right]
\end{aligned}
$$

Reduction. We first notice that if $j=i$ then the factor $\left(\left(\mathbf{U}_{0}\right)_{j}-\left(\mathbf{U}_{0}\right)_{i}\right)$ is zero. Besides, we split the integral in the expression of $\mathbf{K}_{i}$ over Cartesian products of segments, and if we denote by $\partial C_{i}^{k}$ one specific edge of $C_{i}$, then the quantity $F\left(\sigma_{i}-\sigma_{j} ; t\right)$ will be zero for all the edges $\partial C_{j}^{l}$ that have no intersection with $\partial C_{i}^{k}$ (see Figure 1), under a reasonable CFL condition. Moreover we may add one computational restriction, which leads to easier implementations.

Hypothesis 1 We only consider edges of cells $C_{j}$ that are neighboring $C_{i}$.

This hypothesis leads to take into account the same neighbors as for the finite volume method and to easier implementations.

Elementary contributions. Considering Hypothesis 1 or not we have

$$
\operatorname{supp}\left(\delta_{\partial C_{j}}\right) \subset \operatorname{supp}\left(\delta_{E}\right) \cup \operatorname{supp}\left(\delta_{E^{\prime}}\right) \cup \operatorname{supp}\left(\delta_{E^{\prime \prime}}\right)
$$

where $E$ is the edge through which we compute the flux. Both other edges, $E^{\prime}$ and $E^{\prime \prime}$, have a point intersection with $E$. As we split the integral in the expression of $\mathbf{K}_{i}$ over Cartesian products of such segments, we notice that 
quantities $\left(\left(\mathbf{U}_{0}\right)_{j}-\left(\mathbf{U}_{0}\right)_{i}\right) \mathbf{n}_{j} \cdot \mathbf{n}_{i}$ and $A\left(\mathbf{n}_{j}\right)\left(\left(\mathbf{U}_{0}\right)_{j}-\left(\mathbf{U}_{0}\right)_{i}\right)$ are constant and we only have to compute two types of elementary contributions

$$
\begin{aligned}
& \mathcal{I}_{0}=\int_{E \times E^{\prime}} \frac{H\left(c^{2} t^{2}-\left|\sigma-\sigma^{\prime}\right|^{2}\right)}{\sqrt{c^{2} t^{2}-\left|\sigma-\sigma^{\prime}\right|^{2}}} d \sigma d \sigma^{\prime}, \\
& \mathcal{I}_{1}=\int_{E \times E^{\prime}} \frac{H\left(c^{2} t^{2}-\left|\sigma-\sigma^{\prime}\right|^{2}\right)\left(\sigma-\sigma^{\prime}\right) \cdot \mathbf{n}_{E}}{\left|\sigma-\sigma^{\prime}\right|^{2} \sqrt{c^{2} t^{2}-\left|\sigma-\sigma^{\prime}\right|^{2}}} d \sigma d \sigma^{\prime} .
\end{aligned}
$$

We may notice easily that these integrals are invariant under rotations and translations in $\mathbb{R}^{2}$, indeed they only involve Euclidean distances. Therefore the computation of the two above integrals only depend on the length of the edges $E$ and $E^{\prime}$ and of the angle $\alpha$ between them. Besides the CFL condition will imply that only $\mathcal{I}_{0}$ in the case when $E=E^{\prime}$ will depend on the length of $E$ denoted by $\ell$.

The computations are very tedious and details may be found in [2]. The results are however rather simple, namely:

\begin{tabular}{|c|c|c|c|}
\hline$\alpha$ & $0\left(E=E^{\prime}\right)$ & general case & flat \\
\hline $\mathcal{I}_{0}$ & $\ell \pi-2 c t$ & $c t \frac{\pi-|\alpha|}{|\sin \alpha|}$ & $c t$ \\
\hline $\mathcal{I}_{1}$ & 0 & $\frac{\pi}{2} \frac{1+\cos \alpha}{|\sin \alpha|}$ & 0 \\
\hline
\end{tabular}

where the flat angle case is the limit $\alpha \rightarrow \pi$ of the general case. These results are valid under the CFL-like condition

$$
c t \leq \min _{\alpha}|\sin \alpha| \min _{\ell \text { adjacent to } \alpha} \ell .
$$

Approximate values for the correction. We define three sets of indices for neighboring edges (which are represented on Figure 2), namely

$$
\begin{aligned}
& N_{i}^{k}=\left\{(j, l) / j \neq i, \partial C_{j}^{l} \cap \partial C_{i}^{k} \neq \emptyset\right\}, \\
& \bar{N}_{i}^{k}=\left\{(j, l) \in N_{i}^{k} / \operatorname{dim}\left(C_{j} \cap C_{i}\right)=1\right\}, \\
& \tilde{N}_{i}^{k}=\left\{(j, l) \in N_{i}^{k} / C_{j} \cap C_{i}=\partial C_{i}^{k}\right\} .
\end{aligned}
$$

The definition of $\bar{N}_{i}^{k}$ and $\tilde{N}_{i}^{k}$ are two different ways to take into account Hypothesis 1 whereas choosing $N_{i}^{k}$ does not lead to any approximation, according to Figure 1. Of course the relation $N_{i}^{k} \supset \bar{N}_{i}^{k} \supset \tilde{N}_{i}^{k}$ holds true. 

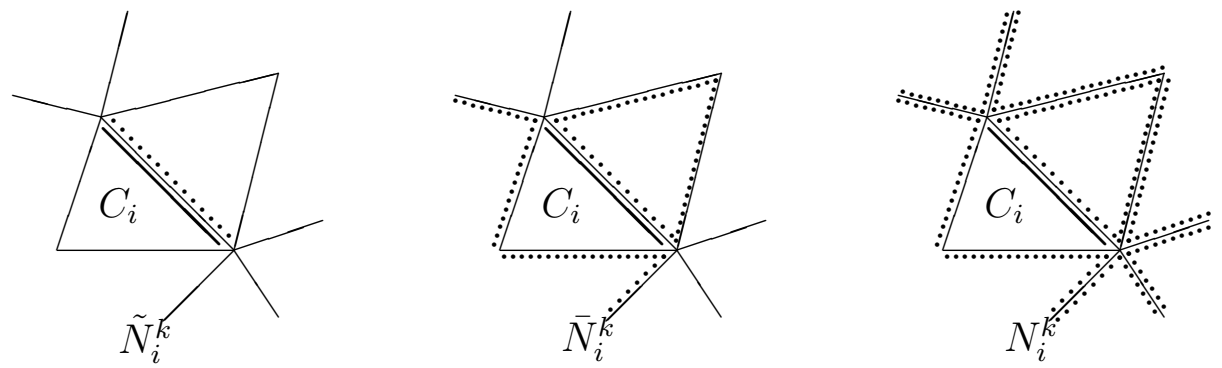

Figure 2: Neighbor sets: $\tilde{N}_{i}^{k}, \bar{N}_{i}^{k}$ and $N_{i}^{k}$. The reference edge $\partial C_{i}^{k}$ is represented with a bold line, edges belonging to the different sets are represented by dotted lines.

Besides $\sharp \bar{N}_{i}^{k}=7$ and $\sharp \tilde{N}_{i}^{k}=1$. Coming back to equation (2.5) we obtain

$$
\begin{aligned}
\mathbf{K}_{i}= & -c^{2} \sum_{k} \sum_{(j, l) \in N_{i}^{k}} \int_{\partial C_{i}^{k} \times \partial C_{j}^{l}}\left[\left(\left(\mathbf{U}_{0}\right)_{j}-\left(\mathbf{U}_{0}\right)_{i}\right) F\left(\sigma_{i}-\sigma_{j} ; t\right)\left(\mathbf{n}_{j} \cdot \mathbf{n}_{i}\right)\right. \\
& \left.+t A\left(\mathbf{n}_{j}\right)\left(\left(\mathbf{U}_{0}\right)_{j}-\left(\mathbf{U}_{0}\right)_{i}\right)\left(\frac{\sigma_{i}-\sigma_{j}}{\left|\sigma_{i}-\sigma_{j}\right|^{2}} F\left(\sigma_{i}-\sigma_{j} ; t\right) \cdot \mathbf{n}_{i}\right)\right] d \sigma_{j} d \sigma_{i} \\
= & -\frac{c}{2 \pi} \sum_{k} \sum_{(j, l) \in N_{i}^{k}}\left(\left(\mathbf{U}_{0}\right)_{j}-\left(\mathbf{U}_{0}\right)_{i}\right)\left(\mathbf{n}_{j} \cdot \mathbf{n}_{i}\right) \mathcal{I}_{0}\left(\partial C_{i}^{k}, \partial C_{j}^{l}\right) \\
& -\frac{c t}{2 \pi} \sum_{k} \sum_{(j, l) \in N_{i}^{k}} A\left(\mathbf{n}_{j}\right)\left(\left(\mathbf{U}_{0}\right)_{j}-\left(\mathbf{U}_{0}\right)_{i}\right) \mathcal{I}_{1}\left(\partial C_{i}^{k}, \partial C_{j}^{l}\right) .
\end{aligned}
$$

If Hypothesis 1 holds true, $N_{i}^{k}$ has simply to be replaced by $\bar{N}_{i}^{k}$ or $\tilde{N}_{i}^{k}$ in all the above expressions. In the special case of boundary elements, we perform exactly the same reconstruction for $\mathbf{K}_{i}$ having previously determined an "external data" which is the subject of Section 3.

Conservation. The fact that $\boldsymbol{\Phi}_{i j}=-\boldsymbol{\Phi}_{j i}$ is called the conservation property for a finite volume scheme. This property holds for the first and higher order schemes discussed in Section 1. We may ask whether this property still holds if we use our corrections. The correction $\mathbf{K}_{i}$ may be written as 


$$
\begin{aligned}
& \mathbf{K}_{i}=- \sum_{j}\left(\boldsymbol{\Phi}_{i j}^{0}+\boldsymbol{\Phi}_{i j}^{1}\right) \text { where } \\
& \boldsymbol{\Phi}_{i j}^{0}=\frac{c}{2 \pi} \sum_{k} \sum_{l /(j, l) \in N_{i}^{k}}\left(\left(\mathbf{U}_{0}\right)_{j}-\left(\mathbf{U}_{0}\right)_{i}\right)\left(\mathbf{n}_{j} \cdot \mathbf{n}_{i}\right) \mathcal{I}_{0}\left(\partial C_{i}^{k}, \partial C_{j}^{l}\right), \\
& \boldsymbol{\Phi}_{i j}^{1}=\frac{c t}{2 \pi} \sum_{k} \sum_{l /(j, l) \in N_{i}^{k}} A\left(\mathbf{n}_{j}\right)\left(\left(\mathbf{U}_{0}\right)_{j}-\left(\mathbf{U}_{0}\right)_{i}\right) \mathcal{I}_{1}\left(\partial C_{i}^{k}, \partial C_{j}^{l}\right) .
\end{aligned}
$$

We notice that we have the "symmetry" properties

$$
\begin{aligned}
& (j, l) \in N_{i}^{k} \quad \Leftrightarrow \quad(i, k) \in N_{j}^{l}, \\
& (j, l) \in \bar{N}_{i}^{k} \quad \Leftrightarrow \quad(i, k) \in \bar{N}_{j}^{l}, \\
& (j, l) \in \tilde{N}_{i}^{k} \quad \Leftrightarrow \quad(i, k) \in \tilde{N}_{j}^{l} .
\end{aligned}
$$

Besides it is clear that $\mathcal{I}_{0}\left(\partial C_{i}^{k}, \partial C_{j}^{l}\right)=\mathcal{I}_{0}\left(\partial C_{j}^{l}, \partial C_{i}^{k}\right)$ and therefore $\boldsymbol{\Phi}_{i j}^{0}=$ $-\boldsymbol{\Phi}_{j i}^{0}$. If we choose neighbors in $\tilde{N}_{i}^{k}$ then the sum only involves one term and $A\left(\mathbf{n}_{j}\right)=-A\left(\mathbf{n}_{i}\right)$. Therefore $\boldsymbol{\Phi}_{i j}^{1}=-\boldsymbol{\Phi}_{j i}^{1}$. For the other choices the expressions for $\mathcal{I}_{1}\left(\partial C_{i}^{k}, \partial C_{j}^{l}\right)$ are quite involved and we do not expect to have the conservation property. This may be one reason to choose the approximation $\tilde{N}_{i}^{k}$ although it is approximate in contrast with $N_{i}^{k}$.

\subsubsection{Higher order case}

Exact value for the correction. Once more we first give an expression for the two terms in equation (2.4). We may now use a value for $\mathbf{U}_{1}$ and the first derivatives of $\mathbf{U}_{0}$ that is constant on each cell. The computations are less tricky in this case:

$$
\begin{aligned}
&\left(F(\cdot ; t) \star \nabla\left(\mathbf{U}_{0}-\left(\mathbf{U}_{0}\right)_{i}\right)\right)\left(\sigma_{i}\right)=\sum_{j / C_{j} \in \mathcal{T}} \int_{C_{j}} F\left(\sigma_{i}-\mathbf{y} ; t\right) \nabla \mathbf{U}_{0}(\mathbf{y}) d \mathbf{y} \\
&=\sum_{j / C_{j} \in \mathcal{T}}\left(\nabla \mathbf{U}_{0}\right)_{j} \int_{C_{j}} F\left(\sigma_{i}-\mathbf{y} ; t\right) d \mathbf{y}, \\
&\left(G(\mathbf{x} ; t) \star \mathbf{U}_{1}\right)\left(\sigma_{i}\right)=-t \sum_{j / C_{j} \in \mathcal{T}} \mathbb{A}\left(\nabla \mathbf{U}_{0}\right)_{j} \int_{C_{j}} \frac{\sigma_{i}-\mathbf{y}}{\left|\sigma_{i}-\mathbf{y}\right|^{2}} F\left(\sigma_{i}-\mathbf{y} ; t\right) d \mathbf{y} .
\end{aligned}
$$




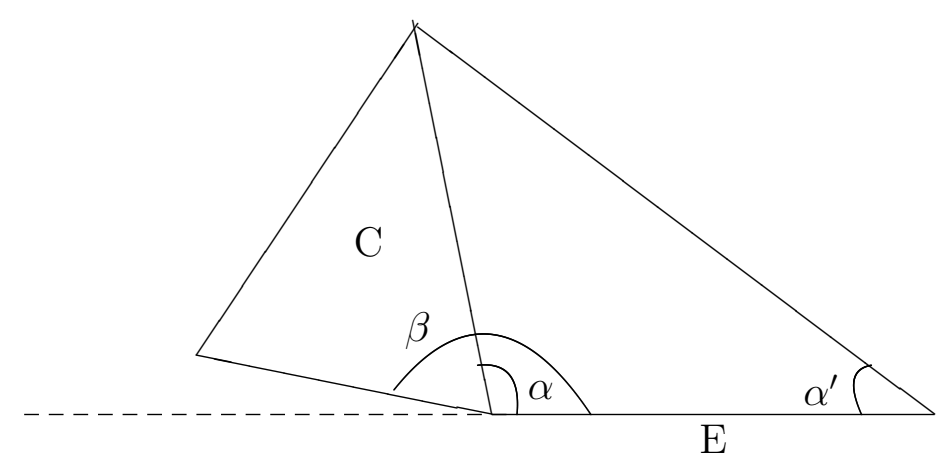

Figure 3: Point-wise intersection case

Therefore

$$
\begin{array}{r}
\mathbf{K}_{i}=c^{2} \sum_{j / C_{j} \in \mathcal{T}} \int_{\partial C_{i} \times C_{j}} d \mathbf{y} d \sigma_{i}\left[\left(\nabla \mathbf{U}_{0}\right)_{j} \cdot \mathbf{n}_{i} F\left(\sigma_{i}-\mathbf{y} ; t\right)\right. \\
\left.-t \mathbb{A}\left(\nabla \mathbf{U}_{0}\right)_{j} \frac{\left(\sigma_{i}-\mathbf{y}\right) \cdot \mathbf{n}_{i}}{\left|\sigma_{i}-\mathbf{y}\right|^{2}} F\left(\sigma_{i}-\mathbf{y} ; t\right)\right] .
\end{array}
$$

Reduction. In this context $j=i$ does not lead to zero contributions. Nevertheless the quantity $F\left(\sigma_{i}-\mathbf{y} ; t\right)$ will be zero for all the cells $\partial C_{j}^{l}$ that have no intersection with $\partial C_{i}^{k}$ (see Figure 1 ). Now a computational restriction might be:

Hypothesis 2 We only treat cells $C_{j}$ that are neighboring $C_{i}$, including $C_{i}$.

Under this condition, given an edge $\partial C_{i}^{k}$, only one cell is taken into account.

Elementary contributions. The first order case, considering or not Hypothesis 1, leads to the computation of two types of contributions concerning two edges. Considering Hypothesis 2 or not will lead to different calculations.

Indeed in the case Hypothesis 2 holds true, the edge $\partial C_{i}^{k}$ always belongs to the cell $C_{j}$ whereas without this hypothesis, $\partial C_{i}^{k}$ and $C_{j}$ may only have a point-wise intersection. This configuration is shown on Figure 3 and obviously

$$
\mathcal{J}_{p}=\left(\mathcal{J}_{p}^{\pi}-\mathcal{J}_{p}^{\alpha}\right)-\left(\mathcal{J}_{p}^{\pi}-\mathcal{J}_{p}^{\beta}\right)=\mathcal{J}_{p}^{\beta}-\mathcal{J}_{p}^{\alpha}, \quad p=0,1
$$


In the case when $\beta$ and/or $\alpha$ are greater than $\pi$, we replace $\mathcal{J}_{p}^{\beta}$ (resp. $\mathcal{J}_{p}^{\alpha}$ ) by $\mathcal{J}_{p}^{\pi-\beta}$ (resp. $\mathcal{J}_{p}^{\pi-\alpha}$ ) in the above expression. This may also easily be generalized to the case when many cells insert between $E$ and $C$.

As in the first order case, we only have to compute two types of elementary contributions

$$
\begin{aligned}
\mathcal{J}_{0} & =\int_{E \times C} \frac{H\left(c^{2} t^{2}-|\sigma-\mathbf{y}|^{2}\right)}{\sqrt{c^{2} t^{2}-|\sigma-\mathbf{y}|^{2}}} d \sigma d \mathbf{y} \\
\mathcal{J}_{1} & =\int_{E \times C} \frac{H\left(c^{2} t^{2}-|\sigma-\mathbf{y}|^{2}\right)(\sigma-\mathbf{y}) \cdot \mathbf{n}_{E}}{|\sigma-\mathbf{y}|^{2} \sqrt{c^{2} t^{2}-|\sigma-\mathbf{y}|^{2}}} d \sigma d \mathbf{y} .
\end{aligned}
$$

The detail of the calculations may be once more found in [2], leading to the following results

\begin{tabular}{|c|c|c|}
\hline$\alpha$ & general case & flat \\
\hline $\mathcal{J}_{0}$ & $\frac{\pi \ell c t}{2}+\frac{\pi}{4} c^{2} t^{2}\left(\left(\frac{\pi}{2}-|\alpha|\right)^{+}-\operatorname{cotan} \frac{|\alpha|}{2}\right)$ & $\frac{\pi \ell c t}{2}-\frac{\pi^{2}}{8} c^{2} t^{2}$ \\
\hline $\mathcal{J}_{1}$ & $-\frac{\pi \ell}{2}+\frac{c t}{2}+\frac{c t}{2}(\pi-|\alpha|) \operatorname{cotan}|\alpha|$ & $-\frac{\pi \ell}{2}$ \\
\hline
\end{tabular}

where $a^{+}=a$ if $a \geq 0$ and $a^{+}=0$ otherwise. To derive these results we have to suppose in addition to condition (2.2.1) that $c t \leq \frac{1}{2} \min l$, which is not a restriction if at least one of the angles in the mesh is smaller than $30^{\circ}$.

We notice that in the point-wise intersection case, the contribution of angle $\alpha^{\prime}$ cancels as well as terms $\frac{\pi \ell c t}{2}$ and $-\frac{\pi \ell}{2}$ in $\mathcal{J}_{0}$ and $\mathcal{J}_{1}$ respectively.

Approximate values for the correction. We define new index sets that determine which cells have to be taken into account once an edge $\partial C_{i}^{k}$ is fixed.

$$
\begin{aligned}
M_{i}^{k} & =\left\{j / C_{j} \cap \partial C_{i}^{k} \neq \emptyset\right\}, \\
\bar{M}_{i}^{k} & =\left\{j \in M_{i}^{k} / \operatorname{dim}\left(C_{j} \cap C_{i}\right) \geq 1\right\}, \\
\tilde{M}_{i}^{k} & =\left\{j \in M_{i}^{k} / C_{j} \cap C_{i} \supset \partial C_{i}^{k}\right\} .
\end{aligned}
$$

These sets also have symmetry properties, for example

$$
\exists k / j \in M_{i}^{k} \Longleftrightarrow \exists l / j \in M_{j}^{l} .
$$


We may now specify the nonzero contributions in the expression (2.7) for $\mathbf{K}_{i}$. First dropping Hypothesis 2

$$
\begin{aligned}
\mathbf{K}_{i}= & c^{2} \sum_{k} \sum_{j \in M_{i}^{k}} \int_{\partial C_{i}^{k} \times C_{j}} d \mathbf{y} d \sigma_{i}\left[\left(\nabla \mathbf{U}_{0}\right)_{j} \cdot \mathbf{n}_{i} F\left(\sigma_{i}-\mathbf{y} ; t\right)\right. \\
& \left.-t \mathbb{A}\left(\nabla \mathbf{U}_{0}\right)_{j} \frac{\left(\sigma_{i}-\mathbf{y}\right) \cdot \mathbf{n}_{i}}{\left|\sigma_{i}-\mathbf{y}\right|^{2}} F\left(\sigma_{i}-\mathbf{y} ; t\right)\right] \\
= & -\frac{c}{2 \pi} \sum_{k} \sum_{j \in M_{i}^{k}}\left(\nabla \mathbf{U}_{0}\right)_{j} \cdot \mathbf{n}_{i} \mathcal{J}_{0}\left(\partial C_{i}^{k}, C_{j}\right) \\
& +\frac{c t}{2 \pi} \sum_{k} \sum_{j \in M_{i}^{k}} \mathbb{A}\left(\nabla \mathbf{U}_{0}\right)_{j} \mathcal{J}_{1}\left(\partial C_{i}^{k}, C_{j}\right) .
\end{aligned}
$$

As in the first order case, Hypothesis 2 consists in replacing $M_{i}^{k}$ by $\bar{M}_{i}^{k}$ or $\tilde{M}_{i}^{k}$ in (2.8).

Conservation In this context, even in the simplest case (choice of $\tilde{M}_{i}^{k}$ for neighboring cells), the computation of $\mathcal{I}_{0}$ takes into account much of the geometry of the neighboring cells. For example $\mathcal{I}_{0}\left(\partial C_{i}^{k}, C_{j}\right)$ involves angles $\alpha$ and $\alpha^{\prime}$ (see Figure 3 ) which belong to $C_{j}$. In the same way, $\mathcal{I}_{0}\left(\partial C_{j}^{l}, C_{i}\right)$ involves angles in $C_{i}$. Therefore no conservation is to be expected.

\subsection{Time discretization}

Taking into account the corrections, the semi-discretized finite volume scheme reads

$$
\left|C_{i}\right| \frac{d \mathbf{U}_{i}}{d t}=-\Psi_{i}^{S W}(\tilde{\mathbf{U}})-\Psi_{i}^{0}(\mathbf{U})(t)-\Psi_{i}^{1}(\mathbf{U})(t),
$$

where the relation with above used notations is $\Psi_{i}^{S W}(\tilde{\mathbf{U}})=\sum_{j} \Phi_{i j}^{S W}\left(\tilde{\mathbf{U}}_{i}, \tilde{\mathbf{U}}_{j}\right)$, $\Psi_{i}^{0}(\mathbf{U})(t)=-\sum_{j} \boldsymbol{\Phi}_{i j}^{0}$ and $\Psi_{i}^{1}(\mathbf{U})(t)=-\sum_{j} \boldsymbol{\Phi}_{i j}^{1}$, using similar notations as in equation (1.6). In this expression $\mathbf{U}_{i}$ and $\mathbf{U}_{j}$ are initial values. In the first order case, $\Psi_{i}^{1}(\mathbf{U})(t)$ is linear with respect to time $t$. On the contrary $\Psi_{i}^{0}$ also contains a constant term for $t>0$ and is zero for $t=0$. It is therefore discontinuous. A way to take this into account is to introduce a parameter $\theta \in[0,1]$ that weights the correction or at least its constant part. Separating 
now the constant and linear parts,

$$
\left|C_{i}\right| \frac{d \mathbf{U}_{i}}{d t}=-\Psi_{i}^{S W}(\mathbf{U})-\Psi_{i}^{C}(\mathbf{U})-t \Psi_{i}^{L}(\mathbf{U}),
$$

may be discretized as

$$
\mathbf{U}_{i}^{n+1}=\mathbf{U}_{i}^{n}-\frac{\Delta t}{\left|C_{i}\right|} \Psi_{i}^{S W}\left(\mathbf{U}^{n}\right)-\frac{\theta \Delta t}{\left|C_{i}\right|} \Psi_{i}^{C}\left(\mathbf{U}^{n}\right)-\frac{\Delta t^{2}}{2\left|C_{i}\right|} \Psi_{i}^{L}\left(\mathbf{U}^{n}\right) .
$$

Parameter $\theta$ has to be chosen relatively small in order to avoid extra dissipation (see [3]). The higher order case may be treated in the same way using a Runge-Kutta method. In this case there are no constant terms or discontinuities to deal with.

\section{Boundary conditions}

All the above study has been performed taking into account no boundary. Since the computational domain has to be bounded by physical or artificial boundaries we have to treat this case. The finite velocity of solutions together with the CFL condition (2.2.1) tell us that we only have to treat the case of boundary cells, the other cells behaving like cells in the whole space. Moreover, we do want to treat the boundary cells as the inner cells for implementation reasons. Therefore to treat different boundary conditions properly, we denote by $L$ the fictitious cell that is symmetric to the boundary cell $K$ with respect to the boundary, and want to define at each time step a value $\mathbf{U}^{L}$ on this fictitious cell in terms of $\mathbf{U}^{K}$. This is useful only in two cases: the case of a reduced number of neighboring cells (Hypotheses 1 and 2) and if the mesh consists in quadrangles.

In this section results will be expressed in terms of the outgoing unit normal vector to the domain $\mathbf{n}=\left(n_{x}, n_{y}, 0\right)$ and the left eigenvectors $\left(l_{-c}, l_{0}, l_{c}\right)$ (resp. the right eigenvectors $\left(r_{-c}, r_{0}, r_{c}\right)$ ) which, up to some normalization, are the lines of $P^{-1}$ (resp. the columns of $P$ ) (see Section 1.2.1).

\subsection{Absorbing conditions}

There are some perfectly absorbing boundary conditions but these conditions are nonlocal and therefore difficult to implement exactly. We nonetheless know local conditions that approximate in a reasonable way these exact 
conditions for Maxwell equations (see [12]). Here we use the first order SilverMüller absorbing condition:

$$
\mathbf{n} \wedge \mathbf{E}^{b}=-\sqrt{\frac{\mu}{\varepsilon}} \mathbf{n} \wedge\left(\mathbf{n} \wedge \mathbf{H}^{b}\right)
$$

where $\mathbf{E}^{b}$ and $\mathbf{H}^{b}$ are respectively the values of $\mathbf{E}$ and $\mathbf{H}$ on the boundary. In order that interference reflections do not interact with the diffracting object, it is sufficient to set the fictitious domain boundary at least two wavelengths away from the object. This restriction is quite stringent for $3 \mathrm{D}$ simulations but quite tractable in $2 \mathrm{D}$.

In terms of the variable $\mathbf{U}^{b}$, condition $(3.1) \operatorname{reads} l_{-c}(\mathbf{n}) \cdot \mathbf{U}^{b}=0$. For the first order Steger-Warming decomposition, such a conclusion is sufficient and the numerical flux across the boundary is simply taken as $A(\mathbf{n}) \mathbf{U}=$ $l_{c}(\mathbf{n}) \cdot \mathbf{U}^{K} r_{c}(\mathbf{n})$. For higher order methods and for our correction we need the full value of $U^{L}$ and two more conditions are needed. They are numerical conditions: the computation within the domain (in $K$ ) yields the two missing information. The characteristic boundary condition method consists in taking these information along in-going (or possibly flat) characteristics.

$$
\begin{aligned}
l_{0}(\mathbf{n}) \cdot \mathbf{U}^{b} & =l_{0}(\mathbf{n}) \cdot \mathbf{U}^{K}, \\
l_{c}(\mathbf{n}) \cdot A(\mathbf{n}) \mathbf{U}^{b} & =l_{c}(\mathbf{n}) \cdot A(\mathbf{n}) \mathbf{U}^{K} .
\end{aligned}
$$

The first numerical condition is chosen in this way because the more natural operator $l_{0}(\mathbf{n}) \cdot A(\mathbf{n}) \mathbf{U}$ is identically zero. The three conditions give a unique value for $\mathbf{U}^{b}$ :

$$
\begin{aligned}
U_{1}^{b} & =\frac{1}{2}\left(\left(1+n_{x}^{2}\right) U_{1}^{K}+n_{x} n_{y} U_{2}^{K}-n_{y} Y U_{3}^{K}\right), \\
U_{2}^{b} & =\frac{1}{2}\left(n_{x} n_{y} U_{1}^{K}+\left(1+n_{y}^{2}\right) U_{2}^{K}+n_{x} Y U_{3}^{K}\right), \\
U_{3}^{b} & =\frac{1}{2}\left(-n_{y} Z U_{1}^{K}+n_{x} Z U_{2}^{K}+U_{3}^{K}\right),
\end{aligned}
$$

which may be summarized as

$$
\mathbf{U}^{b}=\mathbf{U}^{K}+\frac{1}{c} A^{-}(\mathbf{n}) \mathbf{U}^{K} .
$$


As we stressed above, we are interested in the value on the fictitious exterior cell and not in the value on the boundary. The characteristics method implies that we look for $\mathbf{U}^{L}$ such that

$$
\begin{aligned}
l_{-c}(\mathbf{n}) \cdot A(\mathbf{n}) \mathbf{U}^{b} & =l_{-c}(\mathbf{n}) \cdot A(\mathbf{n}) \mathbf{U}^{L} \\
l_{0}(\mathbf{n}) \cdot \mathbf{U}^{b} & =l_{0}(\mathbf{n}) \cdot \mathbf{U}^{L} \\
l_{c}(\mathbf{n}) \cdot A(\mathbf{n}) \mathbf{U}^{b} & =l_{c}(\mathbf{n}) \cdot A(\mathbf{n}) \mathbf{U}^{K}
\end{aligned}
$$

The third equation does not involve $\mathbf{U}^{L}$, and this is expected since the characteristics method does not use this component of $\mathbf{U}^{L}$. To close the system, we choose $l_{c}(\mathbf{n}) \cdot A(\mathbf{n}) \mathbf{U}^{b}=l_{c}(\mathbf{n}) \cdot A(\mathbf{n}) \mathbf{U}^{L}$. Thus we have

$$
\mathbf{U}^{L}=l_{0}(\mathbf{n}) \cdot \mathbf{U}^{K} r_{0}(\mathbf{n})+l_{c}(\mathbf{n}) \cdot \mathbf{U}^{K} r_{c}(\mathbf{n})=\mathbf{U}^{b} .
$$

\subsection{Incident field condition}

We want the incoming field to be the incident field $\mathbf{U}^{i n c}$ on the boundary. The natural condition is

$$
l_{-c}(\mathbf{n}) \cdot \mathbf{U}^{b}=l_{-c}(\mathbf{n}) \cdot \mathbf{U}^{i n c} .
$$

Besides we add the same two numerical conditions as for absorbing boundary conditions and the relation $\mathbf{U}^{L}=\mathbf{U}^{b}$ always holds. Therefore

$$
\left\{\begin{aligned}
U_{1}^{L} & =\frac{1}{2}\left(\left(1+n_{x}^{2}\right) U_{1}^{K}+n_{y}^{2} U_{2}^{i n c}+n_{x} n_{y}\left(U_{2}^{K}-U_{2}^{i n c}\right)-n_{y} Y\left(U_{3}^{K}-U_{3}^{i n c}\right)\right), \\
U_{2}^{L} & =\frac{1}{2}\left(n_{x} n_{y}\left(U_{1}^{K}-U_{1}^{i n c}\right)+\left(1+n_{y}^{2}\right) U_{2}^{K}+n_{x}^{2} U_{2}^{i n c}+n_{x} Y\left(U_{3}^{K}-U_{3}^{i n c}\right)\right), \\
U_{3}^{L} & =\frac{1}{2}\left(-n_{y} Z\left(U_{1}^{K}-U_{1}^{i n c}\right)+n_{x} Z\left(U_{2}^{K}-U_{2}^{i n c}\right)+\left(U_{3}^{K}+\mathbf{U}_{3}^{i n c}\right) .\right.
\end{aligned}\right.
$$

\subsection{Perfectly conducting surface}

The perfectly conducting surface condition is:

$$
\mathbf{n} \wedge \mathbf{E}^{b}=0
$$

We add the same two numerical conditions as for the previous conditions as well as the relation $\mathbf{U}^{L}=\mathbf{U}^{b}$. Therefore

$$
\left\{\begin{aligned}
U_{1}^{L} & =n_{x}^{2} U_{1}^{K}+n_{x} n_{y} U_{2}^{K} \\
U_{2}^{L} & =n_{x} n_{y} U_{1}^{K}+n_{y}^{2} U_{2}^{K} \\
U_{3}^{L} & =-n_{y} Z U_{1}^{K}+n_{x} Z U_{2}^{K}+U_{3}^{K}
\end{aligned}\right.
$$




\section{Conclusion}

We have been able to develop multidimensional corrections to finite volume schemes and to give their explicit value in terms of simple functions which is of importance in view of the numerical implementation compared to the double or triple integrals in their initial formulation. The multidimensional approach takes into account the structure of the equations more than MUSCL (van Leer [25]) approach does. We think that this will lead to more physical solutions when real complex test cases will be run.

These results are of course a first step towards a study which would include charge and current densities, and more complex media. Future developments for this study first include the generalization to heterogeneous media in order to treat aircraft coatings for example. In this context each component of the field is not a solution to a wave equation with uniform light velocity, therefore an extension of our method has to be derived. A careful study of which material constants $\varepsilon$ and $\mu$ have to be considered has to be performed as in the case of the finite volume schemes [21].

The generalization to three-dimensional Maxwell equations may also be addressed but all computation for the corrections have to be reproduced since the kernel $F$ of the three-dimensional wave equation is different.

\section{Acknowledgments}

We thank Dr Bruno Stoufflet (Dassault-Aviation) for his suggestion to use Kirchoff formulae in the context of Maxwell equations.

\section{References}

[1] R. Abgrall Approximation du problème de Riemann vraiment multidimensionnel des équations d'Euler par une méthode de type Roe I and II. C. R. Acad. Sci. Paris, Série I, 319, 499-504 and 625-629 (1994)

[2] B. Bidégaray Calcul formel de corrections multi-dimensionnelles pour les schémas volumes-finis collocalisés pour les équations de Maxwell. Technical report MIP 01.13 (2001)

http://mip.ups-tlse.fr/publi/rapp01/01.13.html 
[3] B. Bidégaray and J.-M. Ghidaglia Second order corrections to the finite volume upwind scheme for the 2D Maxwell equations. in Finite Volumes for Complex Applications II, Problems and Perspectives. R. Vilsmeier, F. Benkhaldoun, D. Hänel eds, 483-490, Hermes (1999)

[4] F. Bonnet, M. Bostan, and L. Fezoui Etude d'une classe de $\beta$ - $\gamma$ schémas en formulation volumes finis pour des problèmes hyperboliques. Technical report INRIA 3288 (1997)

[5] S. Chaïra Sur la résolution numérique des équations d'Euler de la dynamique des gaz par des schémas multidimensionnels. PhD thesis, ENS Cachan (1995)

[6] J.-P. Cioni Résolution numérique de type volumes finis pour la résolution d'équations hyperboliques. $\mathrm{PhD}$ thesis, University Nice Sophia-Antipolis (1995)

[7] J.-P. Cioni, L. Fezoui, and H. Steve A parallel time-domain Maxwell solver using upwind schemes and triangular meshes. Impact Comput. Sci. Engrg, 5, 215-247 (1993)

[8] J.-P. Cioni and M. Remaki Comparaison de deux méthodes de volumes finis en électromagnétisme. Technical report INRIA 3166 (1997)

[9] A. Dervieux Steady Euler simulations using unstructured meshes. Von Karman Institute Lecture Series 85-04 (1985)

[10] J.A. Desideri, A. Goudjo, and V. Selmin Third order numerical schemes for hyperbolic problems. Technical report INRIA 607 (1987)

[11] J. Durlofski, S. Osher, and B. Engquist Triangle based TVD scheme for hyperbolic conservation laws. Technical report ICASE 90-10 (1990)

[12] B. Engquist and A. Majda Radiation Boundary Conditions for Acoustic and Elastic Wave Calculations. Commun. Pure Appl. Math., 32, 313357 (1979)

[13] H. Gilquin and J. Laurens Problèmes de Riemann multidimensionnels pour les systèmes hyperboliques linéaires. Technical report (1991) 
[14] H. Gilquin, J. Laurens, and C. Rosier The explicit solution of the bidimensional Riemann problem for the linearized gaz dynamics equations. Technical report, ENS de Lyon, UMPA 133 (1994)

[15] G. Gimonet, J.-P. Cioni, L. Fezoui, and F. Poupaud Approximation numérique des équations de Maxwell en milieu hétérogène monodimensionnel par un solveur de Riemann exact. Technical report CERMICS 95-37 (1995)

[16] P.D. Lax, A. Harten, and B. Van Leer On the upstream differencing and Godunov type schemes for hyperbolic conservation laws. SIAM Review, 25 (1983)

[17] R. Löhner and J. Ambrosiano A finite element solver for the Maxwell equations. GAMNI-SMAI Conference on Numerical Methods for the solution of Maxwell Equations, Paris (1989)

[18] M. Lukáčová-Medviďová and G. Warnecke Lax-Wendrov type second order evolution Galerkin methods for multidimensional hyperbolic systems. East-West J. Numer. Math., 8, 127-152 (2000)

[19] M. Lukáčová-Medviďová, K.W. Morton, and G. Warnecke Evolution Galerkin methods for hyperbolic systems in two space dimensions. Math. Comput., 69, 1355-1384 (2000)

[20] M. Lukáčová-Medviďová, K.W. Morton, and G. Warnecke Evolution Galerkin Methods for Multidimensional Hyperbolic Systems. ENUMATH'98, World Scientific, 445-452 (1998)

[21] M. Remaki, L. Fezoui, and F. Poupaud Un nouveau schéma de type volumes finis appliqué aux équations de Maxwell en milieu hétérogène. Technical report INRIA 3351 (1998)

[22] D.D. Schnack, I. Lottati, Z. Mikic, and P. Satyanarayana A finitevolume algorithm for three-dimensional magnetohydrodynamics on an unstructured, adaptive grid in axially symmetric geometry. J. Comp. Phys., 40, 263-293 (1981)

[23] V. Shankar, W.F. Hall, and A.H. Mohammadian A time-domain differential solver for electromagnetic scattering problems. Proc. IEEE, 77, 709-720 (1989) 
[24] J. Steger and R.F. Warming Flux vector splitting for the inviscid gas dynamic with applications to finite-difference methods. J. Comp. Phys., 140, 71-121 (1998)

[25] A. van Leer A second-order sequel to Godunov's method. J. Comput. Phys., 32, 101-136 (1979) 